\title{
Effect of Strumming Manipulation in Pain Parameters in Myofascial Pain Syndrome of the Para-scapular Region in Males of the Age Group of 20-30 Years
}

\author{
A M R Suresh ${ }^{1}$, Mohan Kumar Muniyan ${ }^{1 *}$, Kalpana ${ }^{2}$, \\ Tapas Priyaranjan Behera ${ }^{3}$, Ebenezer Wilson Rajkumar. D4, \\ Anoop Kumar Tarsolia', Dimple Kashyap ${ }^{6}$
}

\begin{abstract}
${ }^{1}$ Sr. Physiotherapist, Pandit Deendayal Upadhyay National Institute for the Persons with Physical Disabilities (Divyangjan), New Delhi, India.

${ }^{1 *}$ Incharge-Physiotherapy Deptt., Brookfield Hospital, Bengaluru, India.

${ }^{2}$ Physiotherapist, Manipal Hospital, Old Airport road, Bengaluru, India.

${ }^{3}$ Prosthetist and Orthotist, Pandit Deendayal Upadhyay National Institute for the Persons with Physical Disabilities (Divyangjan), New Delhi, India.

${ }^{4}$ Sr. Occupational Therapist, Pandit Deendayal Upadhyay National Institute for the Persons with Physical Disabilities (Divyangjan), New Delhi, India.

${ }^{5}$ Demonstrator Physiotherapist, Pandit Deendayal Upadhyay National Institute for the Persons with Physical Disabilities (Divyangjan), New Delhi, India.

${ }^{6}$ Physiotherapist, Deptt. of Physical Medicine and Rehabilitation, Kalawati Saran Children's Hospital, New Delhi, India.
\end{abstract}

\begin{abstract}
Background: Treatment of myofascial trigger points can be considered a promising approach for the treatment of patients with myofascial pain syndrome. It would be worthwhile to identify predictors/means of successful myofascial trigger point treatment and to investigate whether the treatment is more successful when used alone or combined such as electrotherapy, thermotherapy and manual therapy. Previous studies have examined the effect of single ischemic compression and a combination of ischemic compression and stretching and concluded that both interventions had positive effects on patients' recovery. However the effects of single component of deep tissue massage such as a brief strummimg manipulation in pain parameters is not understood completely in clinical decision making.

Objective: To study the effects of brief strumming manipulation in pain parameters in myofascial pain of the para-scapular region in males of the age group of 20-30 years.

Materials and Methods: Experimental study design with 25 male participants were recruited on the basis of inclusion and exclusion criteria for the study, active trigger point either over the rhomboid or levator scapulae was identified and marked as per diagnostic criteria described by Simon DG (1999 and 2002). Baseline readings for VAS and PPT were recorded on day one before the intervention and final readings were taken 10 minutes after the sixth session ( 3 days a week on alternate day basis for two weeks). Each treatment session of strumming manipulation was for 2 - 3 minutes followed by a rest period of 2 - 3 minutes and repeated 3 times (total duration of the session was for 12 - 15 minutes) followed by 10 - 15 minutes of Ice compression over the manipulated area. Subjects were instructed to continue ice compression at home 2 - 3 times a day for 10 - 15 minutes each session during the non-interventional days and not to carry out any unaccustomed work like lifting heavy things, straining activities of upper limb during study period. Data collected as pre and post intervention values are analysed statistically.
\end{abstract}


Result: The Pre-VAS mean of $6.88 \pm 0.78$ and the Post-VAS mean of $2.44 \pm 1.29$ and the paired t-test mean difference of $4.44 \pm 1.15$ with $\mathrm{t}(24)=19.17$ with pre and post statistical significance of $\mathrm{p}=0.000$. The Pre-PPT mean of $2.79 \pm 1.13$ and the Post-PPT mean of $4.98 \pm 1.22$ and the paired t-test mean difference of $-2.18 \pm 1.30$ with $t(24)=-8.39$ with pre and post statistical significance of $p=0.000$.

Conclusion: Strumming manipulation followed by ice compression is efficacious in reducing pain and muscle tenderness in male patients with para-scapular region with active MTrPs. Its immediate and short-term effects established in this combination may serve as a prime treatment plan in the clinical setting to counteract pain and muscle tenderness due to active MTrPs.

Key Words: myofascial pain syndrome; trigger points; manipulation; massage; visual analog pain scale; pain threshold.

\section{INTRODUCTION}

Myofascial pain syndrome is collection of the sensory, motor, and autonomic symptoms. Myofascial pain syndromes are known to be caused by trigger points. Trigger points are discrete, focal, hyperirritable spots located in a taut band of skeletal muscle. They produce pain locally and in a referred pattern and often accompany chronic musculoskeletal disorders. Acute trauma or repetitive microtrauma may lead to the development of stress on muscle fibers and the formation of trigger points. Patients may have regional, persistent pain resulting in a decreased range of motion in the affected muscles. These include muscles used to maintain body posture, such as those in the neck, shoulders, and pelvic girdle. Trigger points may also manifest as tension headache, tinnitus, temporo-mandibular joint pain, decreased range of motion in the neck and shoulder ${ }^{[1]}$.

Trigger points develop within muscle sarcomeres. Sarcomeres are the basic building blocks of muscles consisting of actin and myosin myofilaments; muscles move when these myofilaments slide over one another. Trigger points develop when this process becomes attenuated and the sarcomeres becomes overactive; the actin and myosin myofilaments stop sliding over one another. As a result, the sarcomere becomes turned to the permanently 'switched-on' position leading to a state of contraction which leads to muscle hypertonia, weakness, shortening, and fibrosis (muscle stiffness). ${ }^{[2]}$ Trigger points are classified as being active or latent, depending on their clinical characteristics. An active trigger point causes pain at rest. It is tender to palpation with a referred pain pattern that is similar to the patient's pain complaint. This referred pain is felt not at the site of the trigger-point origin, but remote from it. The pain is often described as spreading or radiating. Referred pain is an important characteristic of a trigger point. It differentiates a trigger point from a tender point, which is associated with pain at the site of palpation only. A latent trigger point does not cause spontaneous pain, but may restrict movement or cause muscle weakness. The patient presenting with muscle restrictions or weakness may become aware of pain originating from a latent trigger point only when pressure is applied directly over the point ${ }^{[3-6]}$.

Simon suggested that a therapeutic approach that effectively inactivates tender points should constructively impact the trigger points as well. Methods of treating trigger points include electrotherapy modalities namely (transcutaneous electrical nerve stimulation, ultrasound, low-level laser therapy, microwaves, infrared radiation therapies), local anesthetics (botulinum toxin injection, dry needling), manual therapies (muscle energy technique, ischemic compression technique, spray and stretch technique), myofascial release techniques (strain-counterstrain, integrated neuroinhibitory technique), deep pressure massage, mechanical vibration, thermotherapy-hot packs (moist heat), manipulative therapies, stretching, strengthening exercises, and magnet 
therapies involved in lengthening of shortened or contracted muscle and aid the drainage of fluid or blood, improve the range of motion of a stiff joint, and accentuate the relaxation of the contractile component of the muscles ${ }^{[7-11]}$.

Treating the trigger point by ischemic compression involves applying sustained pressure to the trigger point with sufficient force and for long enough to slow down the blood supply the pressure is gradually applied, maintained, and then gradually released. It is held as long as 60 seconds ${ }^{[12]}$. By myofascial release method, in which both hands are crossed and heels of hands are kept transversely across the top of the tissue with some compressive force, and force is applied with the heels of palm in the cross direction equally for 30 seconds and repeated for 3 times. ${ }^{[13]}$ Frictional massage in parallel direction to fiber orientation for 5-10 minutes 'frees up' scar tissue within a trigger point, allowing muscle fibers to move more normally, increasing blood flow through the tissue and decreasing nerve sensitivity, followed by ice massage with ice cubes for 10-15 minutes is particularly useful in treating pain in small areas irritated by trigger points in muscle, tendon or bursa to cause pain relief ${ }^{[14-15]}$.

Studies had reported beneficial effect of the combination of two manual techniques on managing neck pain and upper trapezius muscle tenderness in male patients with MTrPs ${ }^{[16]}$. However, no studies to date have attempted to reveal the procedure and effectiveness of isolated strumming manipulation in short-term or complete resolution of neck pain and muscle tenderness in active MTrPs. Therefore, the objective of this study was to determine the immediate and short-term effects of brief strumming manipulation in pain parameters in myofascial pain syndrome of in male patients with active MTrPs in levator scapulae and rhomboideus group.

The hypothesis of this study was that there is significant difference between brief strumming manipulation and pain parameters in myofascial pain syndrome and muscle tenderness in male patients with upper trapezius active MTrPs.

\section{METHODS AND MATERIALS Study design and participants}

Experimental (pre and post test) study design with 25 male participants, who reported to Pandit Deendayal Upadhyay National Institute for the persons with physical disabilities (Divyangjan), New Delhi, India with the complaints of pain over the para-scapular region of the dominant arm for a duration of 3-6 months; i.e. Right para-scapular region within the age group of 20-30 years for treatment were recruited on voluntary basis and informed consent was obtained before the study. The inclusion criteria was pain of more than 6 on VAS, not more than one demonstratable active MTrPs in the medial para-scapular region (Rhomboidus or Levator scapulae), no history of high velocity neck or shoulder injury, no treatment (medical or physical therapy) received in past 3-6 months, no history of chronic inflammatory, autoimmune and hereditary diseases. The exclusion criteria was degenerative and acquired shoulder joint disease cervical syndrome, permanent tattoo in the region of cervical and shoulder area, injuries, fractures and surgeries of the neck and shoulder region, known history of oral or injectable medications for more than two weeks, any neurological, cardiac, orthopedic and pulmonary diseases, unwillingness to participate in the study, and any other disease that may interfere with the diagnosis and interventional methods.

\section{Independent Variable}

Strumming manipulation followed by Ice compression

\section{Dependent Variable}

Pain (VAS)

Pain pressure threshold (PPT)

\section{Procedure}

Twenty five male participants were recruited on the basis of inclusion and 
exclusion criteria for the study. Participants were explained in detail about the procedure of strumming manipulation and pain, pressure threshold assessment using VAS (Visual analog scale) and PPT (Pressure pain threshold). Each participant was assessed thoroughly by taking history, examining and performing special tests on cervical spine and shoulder to rule out any other conditions which may interfere with our study. Active trigger point over the Rhomboid or Levator scapulae was identified and marked with a skin marker as per diagnostic criteria as under:

The diagnostic criteria to identify and locate the active MTrPs as described by Simon DG (2002), Gerwin RD (2014), and Fernández-de-las-Peñas C (2017) in their studies ${ }^{[7,17-18]}$. We considered the following five points to identify and differentiate between active and latent MTrPs: presence of (a) a taut band within the muscle, (b) a hypersensitive tender focus in the taut band, (c) spontaneous pain, (d) local twitch response on snapping palpation, and (e) a referred sensation on palpation. We considered the active MTrPs if they fulfilled at least the first three points (a), (b), and (c) and the latent trigger points if they did not fulfill the last two points (d) and (e), thus included and excluded from the study, respectively.

Baseline readings for VAS and PPT were recorded on day one before the intervention and final readings were taken 10 minutes after the sixth session ( 3 days a week on alternate day basis for two weeks). Each treatment session of strumming manipulation was for 2 - 3 minutes followed by a rest period of $2-3$ minutes and repeated 3 times (total duration of the

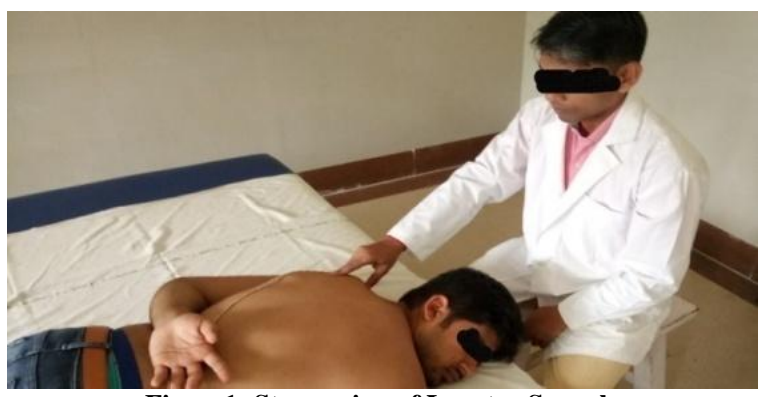

Figure1: Strumming of Levator Scapulae session was for 12 - 15 minutes) followed by 10 - 15 minutes of Ice compression over the manipulated area. Subjects were instructed to continue ice compression at home 2 - 3 times a day for 10 - 15 minutes each session during the non-interventional days and not to carry out any unaccustomed work like lifting heavy things, straining activities of upper limb during study period. Data collected as pre and post intervention values are analysed statistically.

\section{Interventional protocol Strumming manipulation}

Place the patient in a comfortable position, where the affected Levator scapulae and rhomboids muscles can undergo full excursion. Lubricate the skin if required, identify and locate the trigger point or taut band, position the operators thumb/elbow just beyond the taut band, and reinforce with your other hand. This should be experienced by the patient as discomfort and not pain, apply sustained pressure, and continue stroking in the same direction towards the attachment of the taut band, strumming finger runs across the taut bands at the level of the TrPs over the nodules from one side of the muscle to the other, in strumming the operator's finger pulls perpendicularly across the muscle fibers rather than along the length of the fibers ${ }^{[19]}$. The technique is continued for 2 - 3 minutes till a release is palpated or reactive hyperaemia is observed. A rest period of 2 3 minutes is allowed before the next round of strumming manipulation. The entire procedure was repeated three times for a duration of 12 - 15 minutes in total followed by Ice compression for $10-15$ minutes.

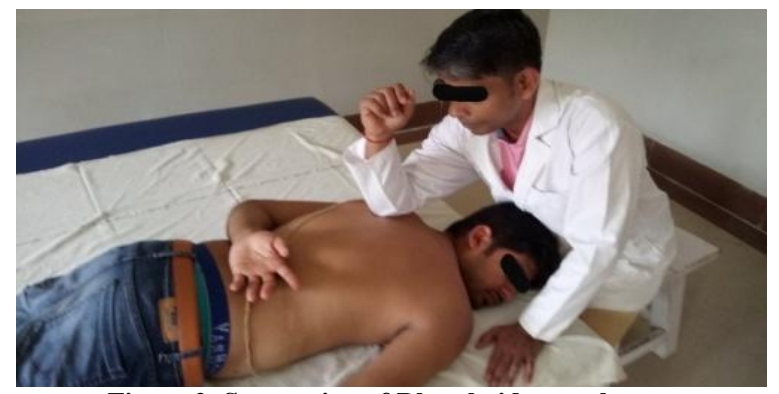

Figure 2: Strumming of Rhomboids muscle. 

para-scapular region in males of the age group of 20-30 years.

\section{Parameters studied}

\section{Visual Analog scale (VAS) ${ }^{[20]}$}

The VAS is a continuous scale comprised of a horizontal line, usually 10 centimetres $(100 \mathrm{~mm})$ in length. The scale is most commonly anchored by "no pain" (score of 0) and "pain as bad as it could be" or "worst imaginable pain" (score of 10). The VAS is self-completed by the respondent. The respondent is asked to place a line perpendicular to the VAS line at the point that represents their pain intensity. Using a ruler, the score is determined by measuring the distance $(\mathrm{mm})$ on the $10-\mathrm{cm}$ line between the "no pain" anchor and the patient's mark, providing a range of scores from 0-100. A higher score indicates greater pain intensity. Based on the distribution of pain, VAS scores in postsurgical patients (knee replacement) who described their postoperative pain intensity as none, mild, moderate, or severe, the following cut points on the pain VAS had been recommended: no pain $(0-4 \mathrm{~mm})$, mild pain (5-44 mm), moderate pain (45-74 $\mathrm{mm})$, and severe pain $(75-100 \mathrm{~mm})$.

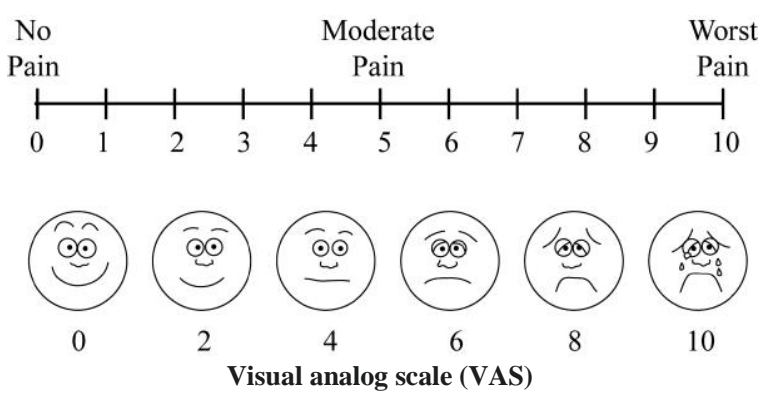

\section{Algometer/Pressure pain threshold (PPT)}

Algometer imply pressure tolerance testing, the maximum amount of pressure one may endure and, by nomenclature the first point at which a pressure sensation is sensed as pain. This is a handheld device and has a "maximum hold" function that displays the maximum pressure obtained in one application. Most commonly, these devices have a $1-\mathrm{cm}^{2}$ pressure application surface and display force readings in Newtons or kilograms of force. It has been noted that the force application should be perpendicular to the body surface, and the rate should be constant at an approximate rate of $1 \mathrm{~kg} / \mathrm{cm}^{-2}$ or $10 \mathrm{~N}$ to increase reliability. Application of force at a faster rate may provoke a low false threshold reading.

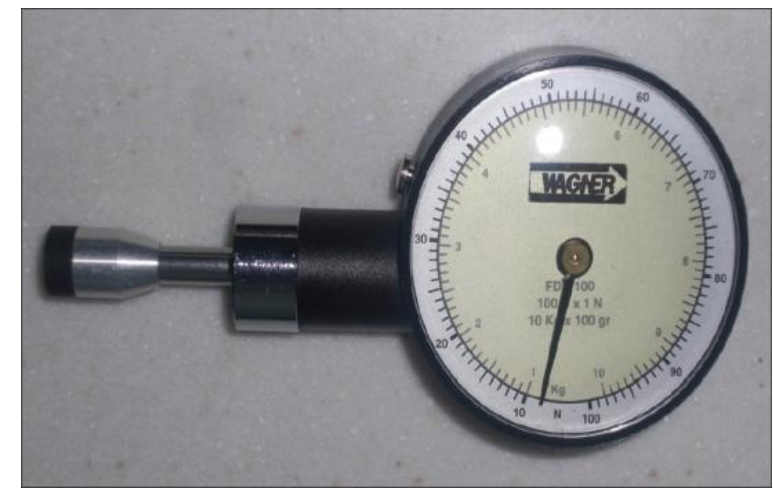

Algometer- Force Dial FDK 20 (Wagner Instruments)

\section{Data analysis \\ Statistical Methods}

Descriptive statistical analysis has been carried out in the present study. Outcome measurements as pre and post VAS and PPT are measured and presented as mean \pm SD. Significance is assessed at 5 $\%$ level of significance with $\mathrm{p}<0.05$ less than this is considered as statistically significant difference.

\section{Statistical tests}

Paired ' $t$ ' test as a parametric test had been used to analysis the variables, pre and post values with $t$-value and $p$ value for the level of significance.

\section{Statistical software} SPSS 20.0

The Statistical software used is

\section{RESULT}

\begin{tabular}{|l|l|l|l|l|l|}
\hline \multicolumn{6}{|c|}{ Table 1: Demography of the subjects- Age, Duration of pain and BMI. } \\
\hline & N & Minimum & Maximum & Mean & Std. Deviation \\
\hline Age in years & 25 & 21 & 29 & 23.84 & 2.688 \\
\hline Duration in Months & 25 & 6 & 20 & 11.68 & 4.059 \\
\hline BMI in $\mathrm{Kg} / \mathrm{m}^{2}$ & 25 & 19.0 & 26.7 & 23.112 & 2.4685 \\
\hline
\end{tabular}


A M R Suresh et.al. Effect of strumming manipulation in pain parameters in myofascial pain syndrome of the para-scapular region in males of the age group of 20-30 years.

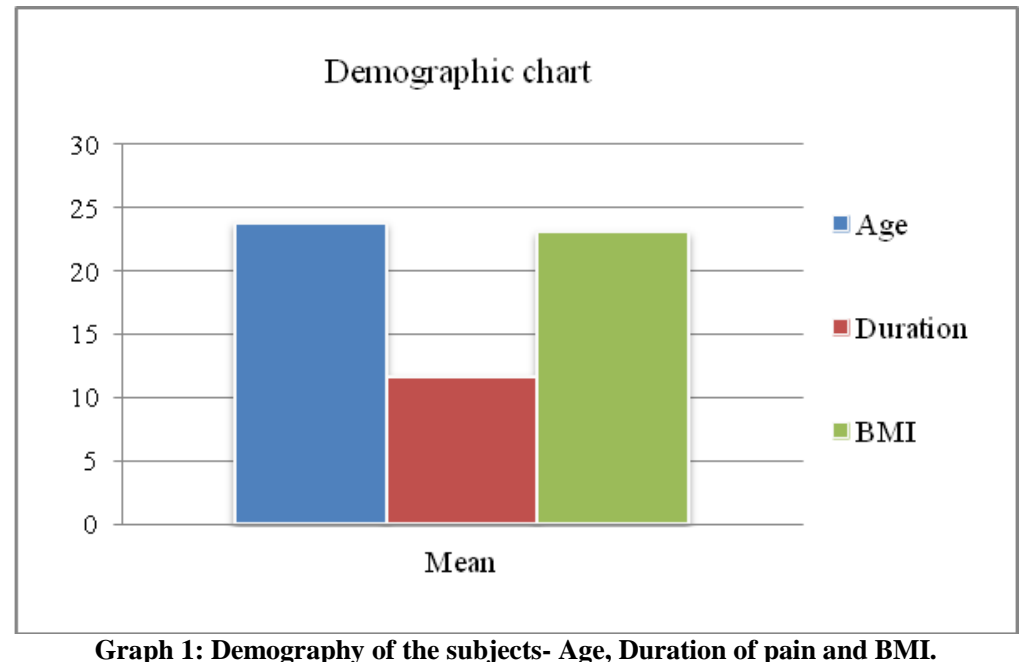

The above table 1 and Graph 1 show that there were 25 subjects with mean age $23.84 \pm 2.68$ years, the mean duration of pain was $11.68 \pm 4.05$ months and the mean BMI was $23.11 \pm 2.46 \mathrm{~kg} / \mathrm{m}^{2}$ in the study.

\begin{tabular}{|l|l|c|c|c|c|}
\hline \multicolumn{5}{|c|}{ Table 2: Comparison of Pre and Post effects on VAS. } \\
\hline & Mean & N & Std. Deviation & Std. Error Mean \\
\hline & Pre VAS & 6.880 & 25 & .7810 & .1562 \\
\cline { 2 - 6 } & Post VAS & 2.440 & 25 & 1.2936 & .2587 \\
\hline
\end{tabular}

\begin{tabular}{|c|c|c|c|c|c|c|c|c|}
\hline & \multicolumn{5}{|c|}{ Paired Differences } & \multirow{3}{*}{$\mathbf{t}$} & \multirow{3}{*}{ Df } & \multirow{3}{*}{$\begin{array}{l}\text { Sig. (2- } \\
\text { tailed) }\end{array}$} \\
\hline & \multirow[t]{2}{*}{ Mean } & \multirow[t]{2}{*}{$\begin{array}{l}\text { Std. } \\
\text { Deviation }\end{array}$} & \multirow{2}{*}{$\begin{array}{l}\text { Std. } \\
\text { Error } \\
\text { Mean }\end{array}$} & \multicolumn{2}{|c|}{$\begin{array}{l}\text { 95\% Confidence Interval of the } \\
\text { Difference }\end{array}$} & & & \\
\hline & & & & Lower & Upper & & & \\
\hline Pre VAS - Post VAS & 4.4400 & 1.1576 & .2315 & 3.9622 & 4.9178 & 19.178 & 24 & 000 \\
\hline
\end{tabular}

The above table shows the Pre-VAS mean of $6.88 \pm 0.78$ and the Post-VAS mean of $2.44 \pm 1.29$ and the paired t-test mean difference of $4.44 \pm 1.15$ with $t(24)=19.17$ with pre and post statistical significance of $\mathrm{p}=0.000$.

\begin{tabular}{|l|c|c|c|c|c|}
\hline \multicolumn{6}{|c|}{ Table 3: Comparison of Pre and Post effects on PPT. } \\
\hline & Mean & N & Std. Deviation & Std. Error Mean \\
\hline & Pre PPT & 2.792 & 25 & 1.1358 & .2272 \\
\cline { 2 - 6 } & Post PPT & 4.980 & 25 & 1.2254 & .2451 \\
\hline
\end{tabular}

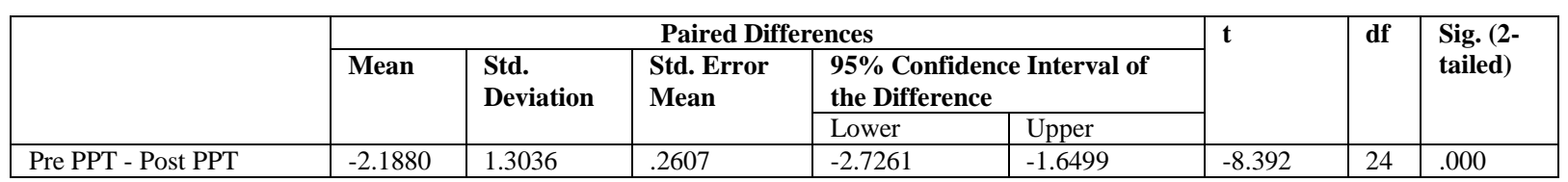

The above table shows the Pre-PPT mean of $2.79 \pm 1.13$ and the Post-PPT mean of $4.98 \pm 1.22$ and the paired t-test mean difference of $-2.18 \pm 1.30$ with $t(24)=-8.39$ with pre and post statistical significance of $\mathrm{p}=0.000$.

\section{DISCUSSION}

According to Simons ${ }^{[13]}$, many of the manual treatment methods stay the same or are only slightly modified (all include some form of mechanical pressure), it is the underlying theory as to why they are effective that continues to evolve with further study. Modalities and manual treatments are often clinically effective for deactivating active MTrPs and desensitizing sensitized spinal segments, and are commonly employed as a first line of treatment before attempting more invasive therapies. The concept of pain relief and decreased muscle tenderness (trigger point sensitivity) by can be understood through its neuro-physiological effect such as inhibitory 
Golgi tendon reflex and descending pathway of pain modulation theories, antiinflammatory and vascular effects ${ }^{[22]}$. At the same time, the mechanoreceptors available in the joint and muscles get activated which further leads to the excitation of sympathetic system via somatic afferent and activation of the periaqueductal gray matter (PEG) which regulate the descending pain modulation ${ }^{[23]}$. Rhythmic muscle compressions also affect the rate of lymphatic and blood flow that bring the changes in interstitial pressure and increase transcapillary blood flow. Vascular blood flow desensitizes the peripheral nociceptive chemical mediators such as cytokines. The effects can be also explained through the concept of lengthening of muscle fibers, which would help dictate the length of the affected soft tissues. Furthermore, there is evolving proof supporting the activation of agonistantagonist inhibitory pathways with the application of manual intervention. Hence, different mechanisms would probably act at the same time to reduce pain intensity and muscle tenderness due to active MTrPs.

The mechanism of the pain relief and reduction of stiffness can be described by the concept of the "barrier release" proposed by Lewit (1991), in which the therapist slowly applies pressure to the MTrPs until a conclusive increase in resistance is perceived, i.e., the barrier, which is usually sensed as not being painful by the subject ${ }^{[24]}$. Hong et al. (1993) proved that prime results in decreasing pain from MTrPs were found with compression techniques used on the deep soft tissue when matching conventional soft-tissue manipulation ${ }^{[25]}$. Thus, the abovementioned studies revealed that the application of compressive manipulations may induce analgesia and improve muscle tenderness (trigger point sensitivity) by the following mechanism. The pressure treatments may cause pain relief as a result of reactive hyperemia in the MTrPs region or act a spinal reflex mechanism for relieving muscle spasms ${ }^{[26]}$. Local pressure may align sarcomere length in the affected MTrPs and thus reduce pain, while deep pressure could offer effective stretching and mobilization of the taut bands ${ }^{[26]}$. Fryer and Hodgson (2005) already proved that local muscle tenderness due to MTrPs decreased only because of a change in tissue sensitivity rather than any unintentional release of pressure by the practitioner ${ }^{[12]}$. Hence, it can be concluded that pressure stretch treatment may be useful for decreasing parascapular pain (VAS) and improving muscle tenderness (PPT) in patients with active MTrPs.

\section{CONCLUSION}

This study validated our hypothesis and concluded that Strumming manipulation followed by ice compression is efficacious in reducing pain and muscle tenderness in male patients with parascapular region active MTrPs. Its immediate and short-term effects established in this combination may serve as a prime treatment plan in the clinical setting to counteract the pain and muscle tenderness due to active MTrPs. The clinical relevance of our findings to practice is that it is highly effective in dismissing MTrPs pain within a very brief period of time, is cost effective, is non-invasive, and achieves relief without causing much pain.

\section{Limitations of the study}

This study was conducted on small sample and included only male participants. The short-term effect on outcome measures as intervention was done only for two weeks, i.e. 6 sessions, the carry over effect of the study has not been studied. Effect on muscle strength and function were not measured. The factors that references on pain such as lifestyle activities, obesity, severity of manifestations and functional limitations were not considered. We propose to conduct a similar study among females and compare the results with current study. For this reason, the result of this study cannot be generalized for the female population of the same conditions. In addition, there was lack of advanced 
technology for measuring amount of pressure required to stretch the muscle fibers/compress the trigger points to neutralize the MTrP pain. Moreover, only the immediate and short-term effect strummimg manipulation was assessed on unilateral para-scapular MTrPs pain and muscle tenderness. Therefore, the abovementioned shortcomings should be addressed by conducting a study on longterm effectiveness (12-week follow-up) of these combination techniques in bilateral para-scapular region active MTrPs pain and muscle tenderness using advanced tools such as isokinetic machine and finger pressure algometer to execute an accurate and definitive amount of muscle contraction and application of pressure, respectively.

Conflict of Interest: The authors declared no conflicts of interest with respect to the authorship and/or publication of this article.

\section{Ethical Approval: Approved.}

Funding: This research received no specific grant from any funding agency in the public, commercial, or not-for-profit sectors.

ACKNOWLEDGEMENT: The authors acknowledge the institution for providing an opportunity to work on the title of the study. The authors are grateful to the subjects that participated in the study. Special thanks to Mr. Pradeep Marandi, Supdt. PT who managed the subjects and study environment for smooth conduction of our study.

Abbreviations: MPS: Myofascial pain syndrome; MTrPs: Myofascial trigger points; PPT: Pain pressure threshold; VAS: Visual analogous scale.

\section{REFERENCES}

1. David J. Alvarez, Pamela G. Rockwell, D.O.et al Trigger Points: Diagnosis and Management Am Fam Physician. 2002 Feb 15; 65(4):653-661.

2. Luime JJ, Koes BW, Hendriksen IJ, et al. Prevalence and incidence of shoulder pain in the general population: A systematic review. Scand J Rheumatology. 2004; 33: 73-81. doi:10.1080/03009740310004667.

3. Hong CZ, Hsueh TC. Difference in pain relief after trigger point injections in myofascial pain patients with and without fibromyalgia. Arch Phys Med Rehabil. 1996;77:1161-6.

4. Han SC, Harrison P. Myofascial pain syndrome and trigger-point management. Reg Anesth. 1997; 22: 89-101.

5. Ling FW, Slocumb JC. Use of trigger point injections in chronic pelvic pain. Obstet Gynecol Clin North Am. 1993; 20:809-15.

6. Fricton JR, Kroening R, Haley D, Siegert R. Myofascial pain syndrome of the head and neck: a review of clinical characteristics of 164 patients. Oral Surg Oral Med Oral Pathol. 1985; 60:615-23.

7. D. G. Simons, "Understanding effective treatments of myofascial trigger points," Journal of Bodywork and Movement Therapies, vol. 6, no. 2, pp. 81-88, 2002.

8. M. Benito-de-Pedro, R. Becerro-de-BengoaVallejo, M. E. Losa-Iglesias et al., "Effectiveness between dry needling and ischemic compression in the triceps surae latent myofascial trigger points of triathletes on pressure pain threshold and thermography: a single blinded randomized clinical trial," Journal of Clinical Medicine, vol. 8, no. 10, p. 1632, 2019.

9. L. Chaitow, Positional Release Techniques, Churchill Livingstone, Elsevier, London, UK, 4th edition, 2015.

10. C.-R. Hou, L.-C. Tsai, K.-F. Cheng, K.-C. Chung, and C.-Z. Hong, "Immediate effects of various physical therapeutic modalities on cervical myofascial pain and trigger point sensitivity," Archives of Physical Medicine and Rehabilitation, vol. 83, no. 10, pp. 1406-1414, 2002.

11. H. Gemmell, P. Miller, and H. Nordstrom, "Immediate effect of ischaemic compression and trigger point pressure release on neck pain and upper trapezius trigger points: a randomised controlled trial," Clinical Chiropractic, vol. 11 , no. 1, pp. 30-36, 2008.

12. Fryer G, Hodgson L. The effect of manual pressure release on myofascial trigger points in the upper trapezius muscle. J Bodywork Movement Ther. 2005; 9: 248-255. doi: 10.1016/j.jbmt.2005.02.002. 
A M R Suresh et.al. Effect of strumming manipulation in pain parameters in myofascial pain syndrome of the para-scapular region in males of the age group of 20-30 years.

13. Simons DG, Travell JG, Simons LS, Travell JG. Travell \& Simons' Myofascial Pain and Dysfunction. The trigger point manual. 2nd edn. Philadelphia, USA: Lippincott Williams and Wilkins. 1999.

14. Green S, Buchbinder R, Hetrick S. Physiotherapy interventions for shoulder pain. Cochrane Database Syst Rev. 2003; CD004258. doi:10.1002/14651858.CD004258.

15. Ejnisman B, Andreoli CV, Soares BG, et al. Interventions for tears of the rotator cuff in adults. Cochrane Database Syst Rev. 2004; CD002758. doi: 10.1002/14651858.CD002758.pub2.

16. Iqbal, H. Ahmed, and A. Shaphe, "Efficacy of muscle energy technique in combination with strain-counterstrain technique on deactivation of trigger point pain," Indian Journal of Physiotherapy and Occupational Therapy- An International Journal, vol. 7, no. 3, pp. 118-123, 2013.

17. R. D. Gerwin, "Diagnosis of myofascial pain syndrome," Physical Medicine and Rehabilitation Clinics of North America, vol. 25, no. 2, pp. 341-355, 2014.

18. Fern'andez-de-las-Peñas and J. Dommerholt, "International consensus on diagnostic criteria and clinical considerations of myofascial trigger points: a Delphi study," Pain Medicine, vol. 19, no. 1, pp. 142-150, 2017.

19. Travell \& simon's Myofascial pain and dysfunction the trigger point manual volume 1 upper half of the body $2^{\text {nd }}$ edition 1988.

20. Alghadir, S. Anwer, A. Iqbal, and Z. Iqbal, "Test-retest reliability, validity, and minimum detectable change of visual analog, numerical rating, and verbal rating scales for measurement of osteoarthritic knee pain," Journal of Pain Research, vol. 11, pp. 851-856, 2018.

21. T. K. Koo, J.-Y. Guo, and C. M. Brown, "Test-retest reliability, repeatability, and sensitivity of an automated deformation controlled indentation on pressure pain threshold measurement," Journal of Manipulative and Physiological Therapeutics, vol. 36, no. 2, pp. 84-90, 2013.

22. G. Fryer, "Muscle energy technique: an evidence-informed approach," International Journal of Osteopathic Medicine, vol. 14, no. 1, pp. 3-9, 2011.

23. I. Faqih, N. Bedekar, A. Shyam, and P. Sancheti, "Effects of muscle energy technique on pain, range of motion and function in patients with post-surgical elbow stiffness: a randomized controlled trial," Hong Kong Physiotherapy Journal, vol. 39, no. 1, pp. 25-33, 2019.

24. K. Lewit, Manipulative Therapy in Rehabilitation of the Locomotor System, Butterworth Heinemann, Oxford, UK, Second edition, 1991.

25. C.-Z. Hong, Y.-C. Chen, C. H. Pon, and J. $\mathrm{Yu}$, "Immediate effects of various physical medicine modalities on pain threshold of an active myofascial trigger point," Journal of Musculoskeletal Pain, vol. 1, no. 2, pp. 3753, 1993.

26. Blikstad and H. Gemmell, "Immediate effect of activator trigger point therapy and myofascial band therapy on nonspecific neck pain in patients with upper trapezius trigger points compared to sham ultrasound: a randomised controlled trial," Clinical Chiropractic, vol. 11, no. 1, pp. 23-29, 2008.

How to cite this article: A M R Suresh, Muniyan MK, Kalpana et.al. Effect of strumming manipulation in pain parameters in myofascial pain syndrome of the para-scapular region in males of the age group of 20-30 years. Int $J$ Health Sci Res. 2021; 11(9): 18-26. DOI: https://doi.org/10.52403/ijhsr.20210903 